S. KOBAYASHI

KODAI MATH. J.

5 (1982), 318-328

\title{
ON ANALYTIC CENTERS OF COMPACT SETS
}

\author{
By SHŌJI KoBAYASHI
}

Introduction. In this paper we are concerned with a certain extremal problem involving second derivatives of bounded analytic functions at $\infty$, anlogous to the well-known extremal problem involving first derivatives that gives rise to the Ahlfors function, or to the Riemann map in the case of simply connected domains.

The concepts of analytic diameter and analytic center were first introduced by Vitushkin $[10,11]$ in the way of developping the theory of rational approximation. In $1974 \mathrm{~F}$. Minsker [8] obtained their several properties mainly for planar continua and presented open problems on analytic diameters and analytic centers of general planar sets. Recently the author and N. Suita [7] investigated certain properties of analytic diameters, analytic centers and the associated extremal problems for planar compact sets, and also offered negative answers to the Minsker's problems except the fourth one which asks whether the set of analytic centers of a planar set is contained in its convex hull. In this paper we answer this Minsker's fourth problem affirmatively under a certain symmetric condition.

In Section 1 we list the definitions and notation which we use throughout this paper. In Section 2 we state preliminary known results as a series of lemmas for the convenience of citation. In Section 3 we prove our main theorems, one of which estimates the size of the analytic centers of a compact set when the compact set is contained in a closed disc, and the other when the compact set lies between two lines. In Section 4 we obtain the set of analytic centers as a exact form for simple symmetric compact sets on a line, which shows that the estimate given in [7, Theorem 2] by the author and Suita is sharp.

1. Definitions and notation. Let $K$ be a compact set in the complex plane $C$. We denote by $D=D(K)$ the unbounded component of the complement $K^{c}$ of $K$. Let $A B(D)$ be the Banach space of all bounded analytic functions in $D$ with the uniform norm $\|\cdot\|_{\infty}$, this is

$$
\|f\|_{\infty}=\sup \{|f(z)|: z \in D\}
$$

Received March 30, 1981 
for every $f \in A B(D)$. Let $\mathcal{A}(K)$ denote the admıssible functions for $K$, which means

$$
\mathcal{A}(K)=\left\{f \in A B(D):\|f\|_{\infty} \leqq 1, f(\infty)=0\right\} .
$$

The analytuc capacity $\gamma(K)$ of $K$ is defined by

$$
\gamma(K)=\sup \left\{\left|f^{\prime}(\infty)\right|: f \in \mathcal{A}(K)\right\} .
$$

It is well known that there exists a unique extremal function $f_{0} \in \mathcal{A}(K)$ with $f_{0}^{\prime}(\infty)=\gamma(K)$, which is called the Ahlfors function ([1], [2], [3]).

By Taylor's theorem, any function $f$ analytic at $\infty$ can be expressed in the form

$$
f(z)=f(\infty)+\frac{f^{\prime}(\infty)}{z-z_{0}}+\frac{\beta\left(f, z_{0}\right)}{\left(z-z_{0}\right)^{2}}+\cdots,
$$

for every $z_{0} \in \boldsymbol{C}$. Here $\beta\left(f, z_{0}\right)$ is given by

$$
\beta\left(f, z_{0}\right)=\frac{1}{2 \pi \imath} \int_{|z|=R} f(z)\left(z-z_{0}\right) d z
$$

for such a large $R>0$ that the disc $\{z:|z|<R\}$ contains $K$. For each compact set $K$ with $\gamma(K)>0$, define

$$
\beta(K, z)=\sup \{|\beta(f, z)| / \gamma(K): f \in \mathcal{A}(K)\},
$$

for every $z \in \boldsymbol{C}$. If $\gamma(K)=0$, define $\beta(K, z)=0$ for every $z \in \boldsymbol{C}$. The analytıc diameter $\beta(K)$ of $K$ is defined by

$$
\beta(K)=\inf _{z \in C} \beta(K, z) .
$$

Any point $z_{0}$ for which $\beta(K)=\beta\left(K, z_{0}\right)$ is called an analytic center of $K$, and we denote by $c(K)$ the set of all analytic centers of $K$. It is known that $c(K)$ is a nonempty compact convex set for any $K$ with $\gamma(K)>0$ ([4], [8]).

It is well known that if $\gamma(K)>0$ then for every $z \in C$ there exists a unique function $f \in \mathcal{A}(K)$ for which $\beta(K, z)=\beta(f, z) / \gamma(K)$ (see for example [3] or [5]). We call such a function the $z$-extremal function and denote it by $f_{z}$. Any $z$ extremal function with $z \in c(K)$ is called a $\beta$-extremal function. The author and Suita [7] showed that there uniquely exists the $\beta$-extremal function while $c(K)$ can contain more than one points (see Lemma 6 in the next section). We denote the $\beta$-extremal function by $f_{*}$.

Since all concepts defined above remains unchanged if we add to $K$ the relatively compact components of $K^{c}$, throughout this paper we assume that $K^{c}$ has no relatively compact components. For any $K$ with $\gamma(K)=0$ the problem which we consider in this paper reduces to an obvious one, so we assume $\gamma(K)$ $>0$ throughout this paper. 
2. Preliminary lemmas. In this section we state several known results on analytic centers as a series of lemmas. Lemma 1 states how analytic diameters and analytic centers depend on conformal maps. For the proof see [7].

LEMma 1. Let $K_{j}(j=1,2)$ be two compact sets. Suppose that there exists a conformal map $\phi$ of $D\left(K_{1}\right)$ onto $D\left(K_{2}\right)$ with an expansion

$$
\phi(z)=z+a_{0}+a_{1} z^{-1}+\cdots
$$

about $\infty$, then

$$
\beta\left(K_{1}\right)=\beta\left(K_{2}\right)
$$

and

$$
c\left(K_{1}\right)+a_{0}=c\left(K_{2}\right),
$$

where by $c\left(K_{1}\right)+a_{0}$ we denote the set $\left\{z+a_{0}: z \in c\left(K_{1}\right)\right\}$.

Lemmas 2, $\cdots, 5$ are proved by Minsker [8];

LEMMA 2. For an arbitrary compact set $K$,

$$
\gamma(K) \leqq \beta(K) \leqq \operatorname{diam} K \text {. }
$$

LEMMA 3. If $K$ is symmetruc with respect to a line, then so are $\beta(K, z)$ and $c(K)$.

LEMMA 4. If $K$ is contained in a closed disc of radius $r$, then

$$
\beta(K) \leqq r
$$

and $c(K)$ is contained in the closed disc of radius $2 r$ with the same center.

LEMMA 5. If $K$ is a continum which contains more than one points, then

$$
\gamma(K)=\beta(K)
$$

and $c(K)$ is a singleton which is contained in the convex hull of $K$.

The author and Suita proved the following ([7, Theorem 1, 4 and 5);

LEMma 6. There exists a unique $\beta$-extremal function $f_{*}$ and

$$
f_{*}^{\prime}(\infty)=0
$$

holds.

Lemma 7. Suppose that $K$ consists of a finite number of mutually disjoint 
continua whose boundary is an analytıc closed Jordan curve. If $z_{0} \in c(K)$, then there exists a function $g$ analytic in a nerghborhood of $\bar{D}$ with $g(\infty)=0$ such that

$$
\frac{1}{2 \pi} \int_{\partial K}\left|z-z_{0}-g(z)\right||d z|=\gamma(K) \beta(K)
$$

and

$$
\frac{1}{\imath} f_{*}(z)\left(z-z_{0}-g(z)\right) d z \geqq 0
$$

along $\partial K$.

LEMMA 8. Suppose that $K$ consists of a finite number of mutually disjoint continua each of which contains more than points, then $c(K)$ is a singleton or a line segument.

3. Main results. Theorem 1 is a sharper version of a Minsker's result Lemma 4 in the preceding section.

THEOREM 1. If $K$ is contained in a closed disc of radius $r$, then $c(K)$ is contained in the open disc of radius $R$ with the same center, where $R=\beta(K)+$ $r\left(1-\gamma^{2}(K) / r^{2}\right)$.

Proof. We may assume that $K$ is contained in the disc $\{z:|z| \leqq r\}$, if necessary by using Lemma 1 . Let $f_{0}$ be the Ahlfors function for $K$, and define

$$
F(z)=\left(\frac{1}{r} z f_{0}(z) \frac{r(K)}{r}\right) /\left(1-\frac{r(K)}{r^{2}}-z f_{0}(z)\right) \text {. }
$$

Then we easily see $F \in \mathcal{A}(K)$ and

$$
F^{\prime}(\infty)=f_{0}^{\prime \prime}(\infty) / 2 r\left(1-\frac{r^{2}(K)}{r^{2}}\right)
$$

by a simple calculation. Therefore we obtain

$$
\left|\frac{1}{2} f_{0}^{\prime \prime}(\infty)\right| \leqq r\left(1-\frac{r^{2}(K)}{r^{2}}\right) r(K),
$$

since $\left|F^{\prime}(\infty)\right| \leqq \gamma(K)$. Suppose $z_{0} \in c(K)$, then we see

$$
\left|\beta\left(f_{0}, z_{0}\right)\right|=\left|\frac{1}{2} f_{0}^{\prime \prime}(\infty)-z_{0} f_{0}^{\prime}(\infty)\right|<\gamma(K) \beta(K)
$$

since $f_{0}$ does not coincide with $f_{*}$. Combining (3.3) and (3.4), we obtain

$$
\left|z_{0}\right|<\beta(K)+r\left(1-\gamma^{2}(K) / r^{2}\right),
$$

as desired. 
Remark. Since we see $\beta(K) \leqq r$ by (2.3) if the hypothesis of Theorem 1 is satisfied, Theorem 1 includes the Minsker's estimate on the size of $c(K)$ stated in Lemma 4.

THEOREM 2. Suppose that $K$ lies between parallel two lines, then at least one of the two components of the plane separated by the two lines which do not contain $K$ contains no analytic centers of $K$.

Proof. By using Lemma 1, if necessary, we may assume that the parallel two lines be $\{z: \operatorname{Re} z=0\}$ and $\{z: \operatorname{Re} z=1\}$. Let $K$ be contained in $\{z: 0 \leqq \operatorname{Re} z \leqq 1\}$. Suppose that there exist $z_{0}, z_{1} \in c(K)$ with

$$
\operatorname{Re} z_{0}<0
$$

and

$$
\operatorname{Re} z_{1}>1 \text {. }
$$

Let $\left\{D_{n}\right\}$ be a regular exhaustion of $D=D(K)$, where "regular" means that the boundary $\partial D_{n}$ of $D_{n}$ consists of a finite number of mutually disjoint closed analytic Jordan curves for each $n$. Let $K_{n}=D_{n}^{c}$, and $f_{n, j}$ be the $z_{j}$-extremal function for $K_{n}$ for $j=0,1$. It is well known that $f_{n,}$ converges to $f_{*}$ uniformly on every compact set of $D$ as $n \rightarrow \infty$, since $z_{j} \in c(K)$ means that $z_{j^{-}}$ extremal function for $K$ coincides with $f_{*}$. By Lemma 7 or Theorem 9.4 of [5], we see that there exists a function $g_{n, j}$ analytic in a neighborhood of $\bar{D}_{n}$ with $g_{n, j}(\infty)=0$ such that

$$
\frac{1}{2 \pi} \int_{\partial D_{n}}\left|z-z_{\jmath}-g_{n, j}(z)\right||d z|=\gamma\left(K_{n}\right) \beta\left(K_{n}, z_{\jmath}\right)
$$

and

$$
\frac{1}{\imath} f_{n, j}(z)\left(z-z_{j}-g_{n, j}(z)\right) d z \geqq 0
$$

along $\partial D_{n}$. By the residue theorem, we see

and

$$
\begin{aligned}
& \frac{1}{2 \pi \imath} \int_{\partial D_{n}}\left(z-z_{0}\right) f_{n, 1}(z)\left(z-z_{1}-g_{n, 1}(z)\right) d z \\
& \quad=z_{0} z_{1} f_{n, 1}^{\prime}(\infty)-\left(z_{0}+z_{1}\right) f_{n, 1}^{\prime \prime}(\infty) / 2+f_{n, 1}^{\prime \prime \prime}(\infty) / 6
\end{aligned}
$$

$$
\begin{aligned}
& \frac{1}{2 \pi \imath} \int_{\partial D_{n}}\left(z-z_{1}\right) f_{n, 0}(z)\left(z-z_{0}-g_{n, 0}(z)\right) d z \\
& \quad=z_{0} z_{1} f_{n, 0}^{\prime}(\infty)-\left(z_{0}+z_{1}\right) f_{n, 0}^{\prime \prime}(\infty) / 2+f_{n, 0}^{\prime \prime \prime}(\infty) / 6
\end{aligned}
$$

By (3.6) and (3.7) there exists a $\delta>0$ such that

$$
\operatorname{Re}\left(z-z_{0}\right) \geqq \delta
$$


and

$$
\operatorname{Re}\left(z-z_{1}\right) \leqq-\delta
$$

if $z \in \partial D_{n}$, for every sufficiently large $n$. Combining (3.8), (3.10) and (3.12), we see

$$
\begin{aligned}
& \operatorname{Re}\left\{z_{0} z_{1} f_{n, 1}^{\prime}(\infty)-\left(z_{0}+z_{1}\right) f_{n, 1}^{\prime \prime}(\infty) / 2+f_{n, 1}^{\prime \prime \prime}(\infty) / 6\right\} \\
& \quad=\operatorname{Re}\left\{\frac{1}{2 \pi i} \int_{\partial D_{n}}\left(z-z_{0}\right) f_{n, 1}(z)\left(z-z_{1}-g_{n, 1}(z)\right) d z\right\} \\
& \geqq \frac{\delta}{2 \pi} \int_{\partial D_{n}}\left|z-z_{1}-g_{n, 1}(z)\right||d z| \\
& \quad=\delta \gamma\left(K_{n}\right) \beta\left(K_{n}, z_{1}\right) .
\end{aligned}
$$

Similarly by (3.9), (3.11) and (3.13), we obtain

$$
\begin{aligned}
& \operatorname{Re}\left\{z_{0} z_{1} f_{n, 0}^{\prime}(\infty)-\left(z_{0}+z_{1}\right) f_{n, 0}^{\prime \prime}(\infty) / 2+f_{n, 0}^{\prime \prime \prime}(\infty) / 6\right\} \\
& \leqq-\delta \gamma\left(K_{n}\right) \beta\left(K_{n}, z_{0}\right) .
\end{aligned}
$$

Letting $n \rightarrow \infty$, we obtain from (3.14)

$$
\operatorname{Re}\left\{-\left(z_{0}+z_{1}\right) f_{*}^{\prime \prime}(\infty) / 2+f_{*}^{\prime \prime \prime}(\infty) / 6\right\} \geqq \delta \gamma(K) \beta(K),
$$

while from (3.15)

$$
\operatorname{Re}\left\{-\left(z_{0}+z_{1}\right) f_{*}^{\prime \prime}(\infty) / 2+f_{*}^{\prime \prime \prime}(\infty) / 6\right\} \leqq-\delta \gamma(K) \beta(K) .
$$

This is a contradiction.

Corollary 1. If $K$ lies on a line, then $c(K)$ lies on the line.

Proof. Consider the case where the two lines coincide in the theorem.

COROLlary 2. If $K$ is symmetric with respect to a point, then $c(K)$ is contained in the convex hull co(K) of $K$.

Proof. By applying Lemma 1, if necessary, we may assume that $K$ is symmetric with respect to the origin. Then we see that $c(K)$ is also symmetric with respect to the origin. Suppose that there exists $z \in c(K)$ with $z \notin c o(K)$, then there exists a line $l$ which separates $z$ and $K$. Set $z_{0}=z$ and $z_{1}=-z$, and let $l$ and $-l$ be the parallel two lines in the theorem.

4. Examples. In [7] the author and Suita offered two types of symmetric compact sets on a line which has more than one analytic centers. In this section we obtain the set of analytic centers of such compact sets in a more exact 
form.

ExAmPle 1. Let $0<a<b$ and $K_{1}=[-b,-a] \cup[a, b]$ be a symmetric compact set which lies on the real line, then

$$
c\left(K_{1}\right)=[-a, a] .
$$

EXAMPLE 2. Let $0<a<b<c<d$ with

$$
b^{2}-a^{2}=d^{2}-c^{2}
$$

and $K_{2}=[-d,-c] \cup[-b,-a] \cup[a, b] \cup[c, d]$, then

$$
c\left(K_{2}\right)=[-a, a] \text {. }
$$

Remark. In [7] it is shown that if $K_{1}$ is such a compact set as mentioned in Example 1 then

$$
\beta\left(K_{1}\right)=(a+b) / 2
$$

and

$$
c\left(K_{1}\right)=[-\delta, \delta]
$$

for some positive number $\delta$. For such a compact set $K_{2}$ as mentioned in Example 2 it is shown by a similar way

$$
\beta\left(K_{2}\right)=2\left(b^{2}-a^{2}\right) /(b-a+d-c)
$$

and

$$
c\left(K_{2}\right)=[-\delta, \delta]
$$

for some positive number $\delta$.

Theorem 2 of [7] states

$$
\operatorname{diam} c(K) \leqq 2 \beta(K)
$$

as for the size of $c(K)$. Since the $\beta$-extremal function $f_{*}$ differs from the Ahlfors function $f_{0}$, we easily see that we can omit the equality in (4.8), that is,

$$
\operatorname{dim} c(K)<2 \beta(K)
$$

holds. On observing (4.1) and (4.4) when $a \nearrow b$, we see that the estimate on the size of $c(K)$ is sharp.

Proof of Examples 1 and 2. We can prove the two examples by a similar way. Let $K$ be such a compact set as mentioned in Examples 1 or 2 . Let $G(z)$ be the Green's function of $D=D(K)$ with a logarithmic singularity at $\infty$, so $G(z)$ is harmonic in $D-\{\infty\}, G(z)=0$ on $\partial D$ and $G(z)-\log |z|$ is harmonic near 
$\infty$. The harmonic conjugate ${ }^{*} G$ of $G$ is locally defined, up to an additive constant, so that the differential

$$
P(z) d z=\frac{1}{\imath} d\left(G(z)+i^{*} G(z)\right)
$$

is globally well-defined in $D$. It is seen that $P(z) d z$ is analytic in $D-\{\infty\}$ and that it has a simple pole at $\infty$ with residue $-i$. As for the boundary $\partial D$ of $D$ we consider it two-ply so that it surrounds $D$, then we obtain

$$
P(z) d z=\frac{\partial^{*} G}{\partial s} d s=\frac{\partial G}{\partial n} d s
$$

along $\partial D$, where $d s$ denotes the arc length and $\partial / \partial n$ the inner normal derivative. Therefore, in paticular, we see

$$
P(z) d z \geqq 0
$$

along $\partial D$.

Let $K_{1}^{2}=\left[a^{2}, b^{2}\right]$ and $K_{2}^{2}=\left[a^{2}, b^{2}\right] \cup\left[c^{2}, d^{2}\right]$. By $K^{2}$ we denote $K_{1}^{2}$ or $K_{2}^{2}$, respectively. Let $G_{2}(z)$ be the Green's function of $D_{2}=D\left(K^{2}\right)$ with a logarithmic singularity at $\infty$. Then we easily see

$$
G(z)=G_{2}\left(z^{2}\right) / 2
$$

and

$$
P(z) d z=z P_{2}\left(z^{2}\right) d z
$$

for $z \in D$, where

$$
P_{2}(z) d z=\frac{1}{\imath} d\left(G_{2}(z)+i^{*} G_{2}(z)\right) .
$$

For any $F \in \mathcal{A}\left(K^{2}\right)$ set $f(z)=F\left(z^{2}\right)$, then $f \in \mathcal{A}(K)$ and $\beta(f, 0)=F^{\prime}(\infty)$. Therefore we see by (1.3), (1.6) and (1.7)

$$
\begin{aligned}
\gamma(K) \beta(K) & =\sup \{|\beta(f, 0)|: f \in \mathcal{A}(K)\} \\
& \geqq \sup \left\{\left|F^{\prime}(\infty)\right|: F \in \mathcal{A}\left(K^{2}\right)\right\} \\
& =\gamma\left(K^{2}\right),
\end{aligned}
$$

since $0 \in c(K)$ by Lemma 3 . Let $f_{*}$ be the $\beta$-extremal function for $K$. Since $f_{*}(z)=f_{*}(-z)$ by symmetry, there is an $F \in \mathcal{A}\left(K^{2}\right)$ such that $f_{*}(z)=F\left(z^{2}\right)$. Therefore we see by (1.3)

$$
\gamma(K) \beta(K)=\beta\left(f_{*}, 0\right)=F^{\prime}(\infty) \leqq \gamma\left(K^{2}\right)
$$

Combining (4.16) and (4.17) we obtain

$$
\gamma(K) \beta(K)=\gamma\left(K^{2}\right)
$$


and

$$
f_{*}(z)=F_{0}\left(z^{2}\right),
$$

where $F_{0}$ is the Ahlfors function for $K^{2}$.

By (4.10), (4.14) and (4.19) we see that the zeros of $f_{*}$ coincides with those of $P(z) d z / z$ with counting multiplicity in $D-\{\infty\}$, since $F_{0}$ and $P_{2}(z) d z$ have the same zeros in $D_{2}-\{\infty\}$ by symmetry (cf. [6], [9]). Applying Lemma 7 (and together with Lemma 1), we see that there exists a function $g_{0}$ analytic in $D$ and continuous on $\bar{D}$ with $g_{0}(\infty)=0$ such that

$$
\frac{1}{2 \pi} \int_{\partial D}\left|z-g_{0}(z)\right||d z|=\gamma(K) \beta(K)
$$

and

$$
\frac{1}{l} f_{*}(z)\left(z-g_{0}(z)\right) d z \geqq 0
$$

along $\partial D$, since $0 \in c(K)$. We see that $(1 / i) f_{*}(z)\left(z-g_{0}(z)\right) d z$ and $P(z) d z$ have the same zeros and poles with counting multiplicity in $D$, since $z-g_{0}(z)$ has a simple zero at 0 by symmetry. They are both nonnegative along $\partial D$ by (4.12) and (4.21), so we obtain by comparing the residue at $\infty$

$$
\frac{1}{\imath} f_{*}(z)\left(z-g_{0}(z)\right) d z=\gamma(K) \beta(K) P(z) d z .
$$

Suppose $z_{1} \in c(K)$, and we show $z_{1} \in[-a, a]$. Again by Lemma 7 there exists $g_{1}$ analytic in $D$ with $g_{1}(\infty)=0$ such that

$$
\frac{1}{\imath} f_{*}(z)\left(z-z_{1}-g_{1}(z)\right) d z \geqq 0
$$

along $\partial D$. By a similar argument which we used above we see that the differential

$$
\begin{gathered}
\frac{1}{\imath} f_{*}(z)\left(z-g_{0}(z)\right) d z-\frac{1}{\imath} f_{*}(z)\left(z-z_{1}-g_{1}(z)\right) d z \\
=\frac{1}{\imath} f_{*}(z)\left(z_{1}-g_{0}(z)+g_{1}(z)\right) d z
\end{gathered}
$$

has the same zeros as $P(z) d z / z$, and hence we obtain by comparing the coefficient

$$
\frac{1}{\imath} f_{*}(z)\left(z_{1}-g_{0}(z)+g_{1}(z)\right) d z=z_{1} \gamma(K) \beta(K) P(z) / z .
$$

Therefore we see by (4.22) and (4.25)

$$
\frac{1}{\imath} f_{*}(z)\left(z-z_{1}-g_{1}(z)\right) d z=\gamma(K) \beta(K)\left(1-z_{1} / z\right) P(z) d z .
$$

Since (4.26) must be nonnegative along $\partial D$ by (4.23), we see 


$$
z_{1} / z \in[-1,1] \text {, }
$$

for any $z \in \partial D$, and hence we obtain

$$
z_{1} \in[-a, a]
$$

as desired.

Next suppose $z_{1} \in[-a, a]$, and we show that $z_{1}$ is an analytic center of $K$. For any $f \in \mathcal{A}(K)$ we see by the residue theorem

$$
\begin{aligned}
\beta\left(f, z_{1}\right) & =\frac{1}{2 \pi \imath} \int_{\partial D} f(z)\left(z-z_{1}\right) d z \\
& =\frac{1}{2 \pi} \int_{\partial D} f(z) \frac{\gamma(K) \beta(K) P(z)}{z f_{*}(z)}\left(z-z_{1}\right) d z,
\end{aligned}
$$

since $P(z) / z f_{*}(z)$ is analytic in $D$. Therefore we see

$$
\begin{aligned}
\beta\left(K, z_{1}\right) & =\sup \left\{\left|\beta\left(f, z_{1}\right)\right| / \gamma(K): f \in \mathcal{A}(K)\right\} \\
& \leqq \frac{1}{2 \pi} \int_{\partial D}\left|f(z) \frac{\beta(K) P(z)}{z f_{*}(z)}\left(z-z_{1}\right)\right||d z| \\
& \leqq \frac{1}{2 \pi} \int_{\partial D} \beta(K)\left(1-z_{1} / z\right) P(z) d z \\
& =\beta(K)
\end{aligned}
$$

since $1-z_{1} / z \geqq 0$ for $z \in \partial D$. Then we obtain $z_{1} \in c(K)$ by (1.7).

5. Concluding remarks. On observing Corollary 2 to Theorem 2 and Examples 1 and 2, it seems likely that for any compact set the set of its analytic centers is contained in its convex hull.

In the proof of Examples 1 and 2 we only used the fact that $K$ is a compact set symmetric with respect to 0 which lies on the real line and that the critical points of the Green's function of $D_{2}=D\left(K^{2}\right)$ with a logarithmic singularity at $\infty$ coincide with the zeros of the Ahlfors function for $K^{2}$ except one at $\infty$. If there exist any other sets with such property as $K^{2}$, we can construct a set $K$ by considering the inverse image by $z^{2}$ such that $c(K)$ is a closed interval symmetric with respect to 0 on the real line which intersects with $K$ only at the terminal points. But we know no other such sets than those we used in Examples 1 and 2 (cf. [6, p. 297]).

\section{REFERENCES}

[1] Ahlfors, L. V., Bounded analytic functions, Duke Math. J. 14 (1947), 1-11.

[2] Ahlfors, L. V., Open Riemann surfaces and extremal problems on compact subregions, Comment. Math. Helv 24 (1950), 100-134. 
[3] Gamelin, T. W., Extremal problems in arbitrary domains, Michigan Math. J., 20 (1973), 3-11.

[4] Gamelin, T. W., Uniform algebras, Prentice-Hall, Englewood Cliffs, N. J. (1969).

[5] Gamelin, T. W., Lectures on $H^{\infty}(D)$, La Plata Notas de Math., No 21 (1972).

[6] Kobayashi, S., Schwarz's lemma in $H_{p}$ spaces, Kōdai Math. Semi. Rep. 27 (1976), 291-299.

[7] Kobayashi, S. and Suita, N., On analytic diameters and analytic centers of compact sets, Trans. Amer. Math. Soc., 267 (1981), 219-228.

[8] Minsker, S., Analytic centers and analytic diameters of planar continua, Trans. Amer. Math. Soc. 191 (1974), 83-93.

[9] Rudin, W., Analytic functions of class $H_{p}$, Trans. Amer. Math. Soc. 78 (1955), 46-66.

[10] Vitushkin, A. G., Necessary and sufficient conditions on a set in order that any continuous function analytic at the interior points of the set may admit of uniform approximation by rational functions, Dokl. Akad. Nauk SSSR 171 (1966), 1255-1258=Soviet Math. Dokl. 7 (1966), 1622-1625.

[11] Vitushkin, A.G., Analytic capacity of sets and problems in approximation theory, Uspehi Mat. Nauk 22 (1967), no. 6 (138), 141-199=Russian Math. Surveys 22 (1967), no. 6, 139-200.

Department of Electric Engineering

Technological University of Nagaoka 